"Am 10. September, dem Tage Ihrer Generalversammlung, hatte der Bezirk Zweibrücken auch eine Versammlung, bei der die ganze Direction zugegen war. Ich brauche Sie nicht ziu versichern, dafs mit der aufrichtigsten Herzlichkeit das Wohl des norddeutschen Apothekervereins, unsers ruhmwürdigen Vorbildes, mit allgemeinem Jubel ausgebracht wurde. - -

\title{
Ehrenmitglieder.
}

Der Herr Hofrath und Medicinalrath Dr. Kerst und der IIerr Medicinalrath Dr. Buddeus in Gotha, Herr Geheime Hofrath Dr. Schlegel in Meiningen, Herr Professor Dr. Wie grman $n$ in Berlin, Herr Professor Dr. Bernhardy in Dreifsigacker, Herr Kreisphysicus Hofrath Dr. Schlüter in Königsberg (Neumark), Herr Dr. Behrendt in Berlin, sowie Herr Dr. Thaulow aus Christiania, haben dem Vereine für ihre Erwählung als Ehrenmilglieder desselben Dankschreiben eingesandt.

\section{Schreiben des Herrn Dr. Lessing in Berlin an den Verein.}

Dem hochverehrten Vereine erlaube ich mir hiermit, bei Gelegenheit seiner diesjährigen Stiftungsfeier zu Bielefeld, mein "Handbuch der Geschichte der Medicin" als einen geringen Beweis meiner Theilnahme an diesem für die Vervollkommnung der pharmaceutischen Wissenschaft so wichtigen Institute, zu übersenden, begleitet von den aufrichtigsten Wünschen für die zukünftige Fortdauer der segensreichen und für die gesammte Natur- und Heilkunde so wohlthätigen Wirksamkeit dieses Vereins. Stets ist die Apothekerkunst eine treue Schwester der ärztlichen Kunst gewesen; ihre Schicksale sind so innig mit einander verflochten, dafs die Geschichte beider Hand in Hand geht, - ein schönes Vorbild für die beiderseitigen Fachgenossen, dafs auch sie in gemeinsamer Hingebung und Pflichterfüllung dem erhabenen Ziele, das ihnen beiden in der Beförderung des Menschenwohls und in der Erwei-

Arch. d. Pharm. Il. Reihe. XVII. Bds. 1. Hft. 
terung wissenschaftlicher Kenntnisse gesetzt ist, nachstreben sollen. Der hochverehrte $A_{\text {pothekerverein }}$ hat durch seine Leistungen nicht wenig zur Lösung dieser schwierigen Aufgabe beigetragen, und ein gleich beherzigungswerthes und folgenreiches Beispiel für alle Zeiten gegeben. Stets war ich bemiht, neben der eigentlich ïztlichen Kunst auch die ihrer befreundeten Schwester in den verschiedenen Momenten ihrer Entwickelung und Ausbildung, in ihrem Wechselverhältnifs zu einander möglichst klar darzustellen, und wenn ich ihr in den bisher von mir geschilderten Zeitperioden eigentlich nur eine mehr fragmentarische Aufmerksamkeit zuwenden konnte, so hoffe ich dagegen in der Fortsetzung meines Werkes, die riesenartigen Fortschritte, welche die Pharmacie in den letzten zwei Jahrhunderten gemacht hat, sowohl an sich und in ihrem Znsammenhange mit den Bereicherungen, welche die Botanik, Chemie, Physik und Materia medica gewann, als in ihrem Einflusse auf die Gestaltung der medicinischen Systeme desto evidenter und ausführlicher in ihr gehöriges Licht setzen zu können, wobei es mir der schönste Lohn sein würde, wenn ich meinen Untersuchungen und historischen Ergebnissen die Aufmerksamkeit der so würdigen Vereinsmitglieder in einigem Grade zuzuwenden im Stande gewesen wäre.

Berlin, den 20. August 1838.

$$
\text { Dr. M. B. Lessing, }
$$

prakt. Arzt, Mitglied der Hufeland. med.chirurg. Ges. zu Berlin, der Ges. für Naturund Heilkunde zu Dresden u. s. w.

\section{Hagen-Bucholz'sche Stiftung.}

Das Vorsteheramt der IIagen-Bucholz'schen Stiftung zeigt hiemit an, dafs die Preise der Stiftung für die prämiirten Abhandlungen der von derselben für 1837 und 1838 aufgegebenen Preisfragen, den Verfassern jener Abhandlungen, den Herren: J. Ko hl, W. Flo to, Fr. Brendeke und E. Voll and übersandt worden sind.

Die zwölfte Preisaufgabe der Stiftung, auf das Jahr 1839, ist folgende:

Die Einwirkungen der basischen Körper auf Zucker sind ewar in den neuesten Zeiten mehrfach Gegenstand 